\title{
Making Conversations Persistent Through Computer Mediation: Coordination in a Safety-Critical Domain
}

\author{
Jesper Kjeldskov and Jan Stage \\ Aalborg University, Department of Computer Science \\ $\{j e s p e r, j a n s\} @ c s . a a u . d k$
}

\begin{abstract}
Coordination is a key activity in complex organizations with distributed actors. In this paper, we focus on conversations about coordination in a specific safety-critical domain: steering a large container ship in and out of a harbour. Based on a thorough ethnographic study of work activities in this domain we have identified communication problems and modelled how conversation about coordination is supported by formalized communication. From this conversation model, we have designed a mobile computer system that makes the conversation persistent by facilitating exchange of pre-defined text messages. We have validated the conversation model and the design of the computer system through development and qualitative evaluation of a functional prototype. The evaluation demonstrated that computer mediation to make conversations persistent is highly relevant to the work task considered. The evaluation also uncovered fundamental limitations in the conversation model, so we discuss how it can be extended.
\end{abstract}

\section{Introduction}

The literature on use of computer systems in safety-critical domains reflects two different but related purposes of such systems: (1) to monitor and control specific objects in the domain and (2) to coordinate activity through conversation between actors that may be physically distributed.

The first purpose, to monitor and control objects in the domain, is often seen as the core functionality of safety-critical systems. A safety-critical system can be defined as a "computer electronic or electromechanical system whose failure may cause injury or death to human beings" (Palanque et al., 1998). Typically, a safety-critical system provides its operators with information about and control of key objects in the domain.

Several researchers have analysed malfunctioning safety-critical systems, e.g. the Ariane 5 accident
(Ladkin, 1998a), the London Ambulance Service breakdown (van den Anker and Lichtveld, 2000), and a number of accidents involving commercial aircraft (Ladkin, 1998b). Official enquiries into such cases often conclude that the operators' actions or nonactions were the direct cause of the breakdown. Yet Perrow (1984) demonstrated convincingly that complex systems are so difficult to control that breakdowns are "normal". The Three Mile Island accident in 1979 was one of his examples of such a complex system. Because of this inherent complexity, it is a mistake to blame the operators and impose more regulations and control on their work. Instead of forcing additional rules and regulations on operators of computerized industrial installations, it has been suggested that better designed user interfaces could prevent errors in complex safety-critical systems.

From a human-computer interaction point of view, the problem is not malfunctioning software as such but human error caused by poorly designed user interfaces. A classical example of this is the British Midland 1989 air crash accident, in which pilots erroneously shut down the only operational engine due to a simple mapping mismatch in the cockpit (Ladkin, 1998b). During the last two decades, this discussion has extended into other high-risk areas such as healthcare and air traffic control (Camp et al., 2000; Fields et al., 1999; Mackay, 1999; van den Anker and Lichtveld, 2000). Designing humancomputer interfaces that avoid such incorrect operation even in stressful situations represents a great challenge for the HCI community (Redmil and Rajan, 1996).

The second purpose of using computer systems in safety-critical domains is to coordinate activity through conversation between actors. Some researchers have analysed such conversations in detail. Heath and Luff (1992) described how personnel in a London Underground control room communicated systematically and coordinated a variety of tasks and activities. Their focus was primarily on different forms of communication between two persons that were present in the control 
room. Patterson et al. (1999) were more directly concerned with the role of technology in communication by showing how 'voice loops', which is an auditory groupware technology, was used to support coordination in the space shuttle mission control.

Coordination has also been analysed and described on a general level as a dimension of cooperative work. Cooperative work can be divided into work and coordination. Work is the individual contributions, and coordination is the additional efforts that are needed to combine the individual contributions into a coherent whole. Coordination is often complex because it is distributed and dynamic. In order to handle this complexity actors often rely on specific coordination mechanisms, which are artefacts that guide and support coordination activities (Schmidt and Simone, 1996). Coordination mechanisms can be descriptive like maps or procedural like scripts. In both cases, coordination involves conversation about or articulation of the cooperative task (Divitini, 1999).

Persistent conversations, unlike spoken conversations, are characterized by leaving an observable trace in the form of text on a computer screen, sound files etc. Thus, persistent conversations can occur both asynchronously as in, for example, email but also allow several people effectively speaking at once without the "lock-step synchrony" of face-to-face talk as in, for example, chat and instant messaging (Erickson and Herring 2005). These properties make persistent conversations highly interesting in domains where the use of spoken communication is limited by bottlenecks and by handling several parallel tracks of conversations.

Exploiting the potentials of persistent conversations require that communication is formalized and modelled. Much research has been carried out within this area. As a recent example, Twitchell and Nunamaker (2005) present a method for evaluating and visualizing persistent conversations by means of speech act profiles, creating visual maps of intentions.

This paper discusses how conversations about coordination can be made persistent through computer mediation. The discussion is based on an empirical study of coordination in the safety-critical domain of steering a large container ship in and out of a harbour. In section 2, we describe how we collected and analysed empirical data from this domain through two field studies. Section 3 describes the safety-critical domain, presents the work task of leaving a harbour, which is the focus of this paper, and provides a list of communication problems identified in the conversations about coordination of this work task. A key problem is the lack of persistence. In section 4, we describe how we analysed these conversations, and we present a conversation model based on the language-action perspective. In section 5 , we explain the design of a mobile computer system that mediates the conversations, thereby making them persistent. Section 6 describes how the computer system and the underlying conversation model were validated through a usability evaluation of a prototype of the system. Finally, section 7 concludes the paper by discussing how the study of this safety-critical domain has generated ideas for extending the language-action perspective on conversations about coordination.

\section{Empirical study}

This paper is based on two field studies of conversation about coordination in a safety-critical domain. These studies involved direct observation, video recordings, and interviews. All data were collected on board Maersk Line container ships of the $\mathrm{K}$ class.

The first field study was carried out over a period of several months in 2000 and 2001 and involved researchers taking part in a number of voyages along the coastline of Europe on board the Maersk Line container ships. During these voyages, data consisting of high quality video and audio recordings and written notes regarding work activities, communication between actors and use of technology during operation of the ship was gathered. This field study is documented in qualitative descriptions of the application domain (Andersen, 2000; Nielsen, 2000). In addition, the processes of departing from and arriving at a harbour were recorded on video capturing overall views of the captain, harbour pilot and officers on the bridge as well as close-up views of their interaction with key instruments. The audio channel captured inter-personal communication on the bridge as well as VHF radio communication with the officers that managed the crews fore and aft. Finally, a person with detailed insight into the application domain transcribed a selection of the video recordings. This transcription amounts to approximately 200 pages (see Figure 4 for an excerpt from this transcription).

The two researchers who conducted the first field study have used the data to inform new interface design for existing maritime instruments (Andersen and May, 2001) and to identify a number of work activities in which the use of mobile computer terminals or devices could be desirable. These included diagnostic and maintenance work in the engine room, surveying the condition of reefers during voyages, locating personnel in case of 
accidents and supporting various distributed collaborative work activities.

The authors of this paper used the video recordings to identify other interesting tasks. We selected arrival at and departure from a harbour as the tasks we would focus on. Based on the video recordings and the overall transcripts, we produced a full transcription of the communication between the relevant actors. This prepared us for the second field study that we carried out on board a container ship from the same class as in the first field study. The data analysis and the second field study were carried out between August 2001 and May 2002.

\section{Coordination in a Safety-Critical Domain}

The steering and control of a large container ship is a safety-critical domain. Operational failure may cause serious injury or extensivematerial damage. We have studied the operation of Maersk Line container ships of the K class. A ship from this class is 347 meters long and 43 meters wide, see figure 1.



Figure 1. Sine Maersk. The numbers indicate positions of cooperating teams

Steering a ship of this size is a complex task, especially when manoeuvring inside a harbour where erroneous actions may result in the vessel running aground or colliding with the quay or nearby ships. In either case, this would cause serious material damage, potentially severe injuries and possible loss of human life.

\subsection{The Task of Leaving the Harbour}

When a ship is mooring at a quay in a harbour, it is secured to bollards ashore with a complex pattern of mooring lines. These mooring lines keep the ship in a nearly fixed position and the number and types used depend on the conditions. There are basically two types of mooring lines: a line and a spring. A line is fastened at a position beyond the ship whereas a spring is fastened along the ship. Both types extend from both the bow and the stern, see figure 2 .

When the ship is ready for departure, the first step in leaving the quay is letting the mooring lines go. However, as physical space in a harbour is narrow and the means for manoeuvring are limited in relation to the precision needed to avoid collisions, all lines cannot be released simultaneously.

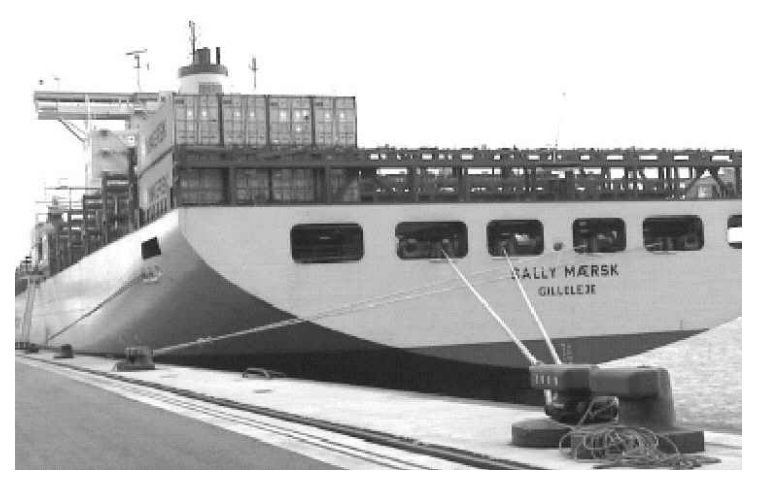

Figure 2. Sally Maersk with stern mooring lines

When a line is let go by releasing it from the bollard and starting to winch it aboard, it will be in the water for a period of time. During this time, no means of propulsion is available due to the risk of the line being sucked in and wrapped around a propeller or thruster. Instead, the vessel can be pulled ahead or astern by means of the remaining lines. Thus lines are released sequentially in accordance with specific needs for manoeuvring.

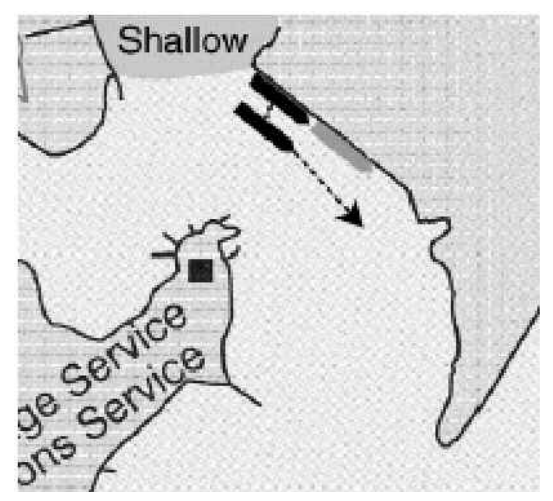

Figure 3. Sally Maersk leaving Felixstowe harbour

One of the cases we studied was Sally Maersk leaving Felixstowe harbour. In this case, there is a shallow area astern and another ship right ahead of her, see figure 3 . First, the mooring lines are reduced to one line and one spring at each end. This is called singling up. Then they reduce further until all lines are released. During this procedure, the ship is also moved sideways away from the quay. This motion involves both the main propeller and the thrusters fore and aft. When all lines are aboard and the ship is far enough away from the quay to clear the other ship, the main propeller is engaged to move the ship forward.

When arriving at the quay, the sequence is more or less reversed. Instead of pulling the lines in, the lines 
are lowered down to the quay, fastened to the bollards and the ship is pulled into a fixed position.

\subsection{Distribution of the Task}

Due to the size of container ships, the work tasks involved when letting go the lines are distributed among a number of actors. The tasks of mooring and letting go the lines involve a number of physically distributed actors at designated locations on the ship:

- Bridge: the captain, chief officers and a local harbour pilot (Position 1 in Figure 1)

- Fore deck: first officer and three assistants (Position 2 in Figure 1)

- Aft deck: second officer and two assistants (Position 3 in Figure 1)

In addition, a number of assistants ashore are involved. In our study, we focussed only on the crew on board the ship.

To insure safety, individual work tasks are carefully coordinated and carried out under strict command of the captain. Thus all coordination and decisions regarding navigation and manoeuvring are made by the captain and the harbour pilot on the bridge and carried out by other actors upon request. On the bridge, the chief officers control the rudder, propeller and thrusters of the ship. Fore and aft, the first and second officers control the winches for rolling out or heaving in the mooring lines via a central remote control.

While the actors on the bridge can see and hear each other, the actors on the deck are out of direct visual and audio contact. Therefore, all conversations between the captain and the officers on deck are transmitted via VHF-based walkie-talkies.

\subsection{Coordinating the Work of the Distributed Actors}

The work of the distributed actors is primarily communicatively coordinated. In order to prevent misunderstandings, the distributed actors refer unconditionally to the commands executed by the captain. Nothing is done, which has not been directly requested by the captain. Drawing on his practical experience with similar operations, the captain and the pilot typically plans the specific sequence of steps to be carried out prior to the operation of mooring or letting go the lines. The plan is then discussed with the officers involved and is revealed in a step-by-step fashion to the actors carrying it out during the operation. This strategy facilitates ad hoc changes by the captain and the harbour pilot adapting to the situation and prevents the teams of distributed actors from getting "out of sync".

At present, all commands are given orally, either directly (on the bridge) or mediated through walkietalkies (to personnel elsewhere on the ship). To verify that a command has been successfully received and understood, the receiver of a command is required to confirm it by repeating the command. If no confirmation is received, the command will be reissued within a given window of time.

In order to carry out the operation of arrival or departure in a safe manner, the captain needs an overview and full control over the propulsion, direction and mooring of the ship. While information about the rudder, propeller and thrusters are available via dedicated instruments on the bridge no information about mooring lines is provided. Maintaining an overview of the ship's mooring thus requires the captain to continuously keep a mental model of the current state of affairs updated, based on conversations in terms of the orders he has given to the crews and the confirmations he has received back.

As this mental model is highly sensitive to errors or misunderstandings in the ongoing communication between bridge and deck, and since disparity between the captain's mental model and the real world may cause wrong decisions to be made, considerable resources are spent on establishing and maintaining common ground (Clark and Schaefer, 1989) among the distributed co-workers.

\subsection{Communication Problems}

In the maritime domain, spoken communication is prevalent and often carries the majority of vital information. To handle this, a set of well-established formalized procedures for communication exists. However, as the size of vessels and the use of technology increases so does the complexity of systems controlling the ship and the cognitive overhead and amount of conversations required for operation. Typically this is supported only by spoken communication mediated through mobile phones, walkie-talkies or closed-circuit intercoms.

On the basis of the empirical material described above, domain experts identified a number of limitations in present means for conversations about coordination on the container ship:

1. Poor sound quality

2. Lack of persistency

3. No possibility for automation

4. Time consuming

5. Language barriers 
6. Bottlenecks

7. Lack of information integration

\subsection{Supporting Persistent Conversations}

The communication problems described above imply that vital information is lost or delayed. Making conversations persistent in this safety-critical domain is, therefore, an interesting area of application for computerized communication and information systems on mobile devices. Recent examples from other safety-critical domains count mobile multimedia communication for telemedicine in emergency ambulance services (van den Anker and Lichtveld, 2000), distributed process control in wastewater plants (Nielsen and Søndergaard, 2000), and capturing of communication in fire-fighting services (Camp et al., 2000).

A possible alternative to the present spoken communication was the use of written text messages on mobile devices. Though this may be cumbersome due to limited performance of handwriting recognition and miniature keyboards, text offers some advantages over voice. Text is a flexible communication channel requiring low cognitive overhead (CSCW, 1994; Popolov et al., 2000). Textual messages are not subject to the ephemeral nature of spoken utterances but are persistent. Furthermore, text-based communication can be done asynchronously and is not influenced by e.g. noise.

While text-based synchronous communication is also time consuming, facilitating selection of predefined standard-phrases as seen on some SMS phones may reduce the time needed for communicating a statement. Predefined standardphrases may also facilitate optional translation between languages. In relation to bottlenecks, applying asynchronous properties to communication by mediating it with a computer system could facilitate parallel information being perceived in sequence rather than simultaneously.

\section{Conversations About Coordination}

In order to determine whether it was possible to make conversations persistent through mediation with mobile computers, we conducted a detailed analysis. This analysis focussed on the conversations that were captured in the transcript and video recordings described in section 2. An excerpt from the complete transcript is shown in Figure 4. This is from the departure from Felixstowe harbour.

Three properties of conversations may be used to identify the representation of textual communication:
1) the aspect and tense, 2) the object and 3) the structure of conversations.

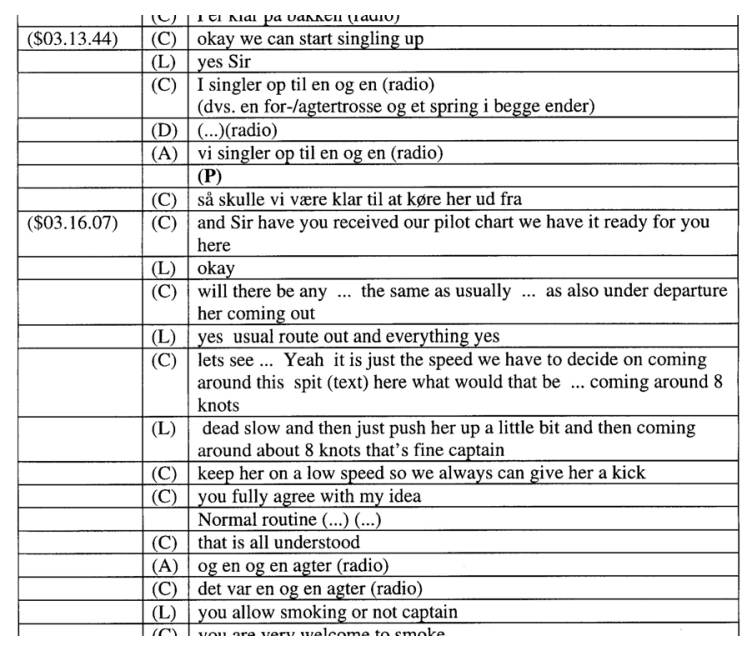

Figure 4. Excerpt from the transcript of conversations

\subsection{Aspect and Tense of Conversations}

On an overall level, a conversation can be categorized by aspect and tense (Andersen, 2000). A conversation is either imminent (future tense), executing (present tense) or ended (past tense). While executing (present) conversations are still open for negotiation, ended conversations imply some kind of mutual agreement having been made among the communicating parties. Imminent (future) conversations are characterized by potentially being initiated when and if appropriate in relation to preceding conversations (ended and executing). In relation to conversation modelling and interface design, this categorization enables us to separate different conversations and differentiate priority. In some situations, ended conversations may be important, while in others only executing tracks are vital.

\subsection{Objects of Conversations}

Communication consisting of a number of interweaved tracks of conversations can be difficult to overview. This can be illustrated with the following extract of the transcription:

$1<$ Captain $>$

$2<1^{\text {st }}$ officer $>$

$3<$ Captain $>$

$4<2^{\text {nd }}$ officer $>$

$5<1^{\text {st }}$ officer $>$

$6<$ Captain $>$ you can let go the bow line

let go bow line

letting go stern spring

bow line let go

bow line let go and you can take the stern spring 
$7<2^{\text {nd }}$ officer $>$

$8<$ Captain $>$

$9<$ Captain $>$

$10<2^{\text {nd }}$ officer $>$

$11<1^{\text {st }}$ officer $>$

$12<$ Captain $>$

$13<2^{\text {nd }}$ officer $>$

$14<$ Captain $>$

and stern spring let go

stern spring let go

you just let go the stern line also

let go line aft

and we have the bow line home

ok

and all let go aft

all let go aft

It is not easy to identify the structure in these conversations. Yet sorting the utterances by the objects of the conversations rather than their sequence, the following structure appears:

$1<$ Captain $>$

$2<1^{\text {st }}$ officer $>$

$5<1^{\text {st }}$ officer $>$

$6<$ Captain $>$

$11<1^{\text {st }}$ officer $>$

$12<$ Captain $>$

$3<$ Captain $>$

$4<2^{\text {nd }}$ officer $>$

$7<2^{\text {nd }}$ officer $>$

$8<$ Captain $>$

you can let go the bow line

let go bow line

bow line let go

bow line let go

and we have the bow line home

ok

$9<$ Captain $>$

$10<2^{\text {nd }}$ officer $>$

$13<2^{\text {nd }}$ officer $>$

$14<$ Captain $>$

and you can take the stern spring

letting go stern spring

and stern spring let go

stern spring let go

you just let go the line aft also

let go line aft

and all let go aft

all let go aft

These conversations are about three interweaved Let go tasks that are conducted by two different teams, represented by the 1st and 2nd officer. They involve two locations: the bow and the stern.

Grouping text in accordance to object rather than sequence thus enables the creation of a more comprehensible representation of conversations as seen in e.g. email and newsgroups. When we are designing for the limited space of a mobile device interface this principle is valuable, as it requires little or no extra space. For a richer representation of sequence, absolute timestamps or timers may be needed.

When we analysed these conversations, we realized that the overall task of letting go the lines follows a pattern of "subtasks" being carried out within a sequence of little variance.

\subsection{Structure of Conversations}

According to Winograd and Flores (1986), the basic course of a conversation for action can be described in a simple diagram with nine different states (Winograd and Flores, 1986, p. 65). Discarding the options of rejection, withdrawal or renegotiation, this diagram can be reduced to five states corresponding to the formalized procedure for communication about execution of a direct command observed in our field studies, cf. Figure 5.

This model applies to each track of conversation in the transcription from the field study above: First, A requests the execution of a task (e.g. "you can let go the bow line"), which B promises to fulfil ("let go bow line"), taking the conversation to state 3. Having fulfilled the request, $\mathrm{B}$ asserts to $\mathrm{A}$ that the execution of the task has been completed ("bow line let go"). Finally, A declares contentment ("bow line let go"), and terminates the conversation (state 5) or goes back to state 3, waiting for additional asserts from B ("and we have the bow line home"). Note that state 1 and 5 in this diagram corresponds to the categories of imminent and ended conversations. State 2 to 4 corresponds to executing conversations.

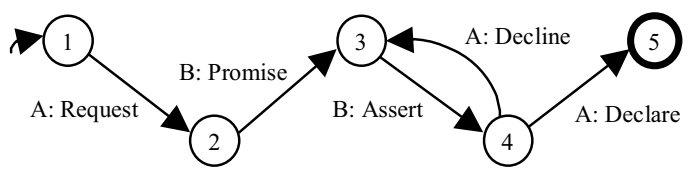

Figure 5. Simplified conversation model

The model of these conversations was achieved by dividing the complete communication with 14 utterances into three separate conversations. Each of these conversations could then be described by the simplified conversation model in Figure 5.

\section{Design of the Mobile Computer System}

Based on the analysis of conversations, we designed a mobile device. The basic design idea was to replace spoken communication with predefined text messages, a graphical representation of an operation in progress, and a filtered list of completed tasks. The system was designed through a number of prototypes. The prototypes were targeted at a Compaq iPAQ handheld computer.

\subsection{Prototypes}

A series of paper-based mockups of possible interface designs were produced on the basis of the analysis described above and inspired by the use of multi-threaded communication and pre-defined messages in other systems. These paper mockups facilitated fundamental discussions of the basic interface design and lead to an overall concept for the user experience. 
On the basis of the paper mockups, detailed design sketches were produced in Microsoft eMbedded Visual Basic. While most of the subsequent refinements of the design were done directly in Visual Basic, larger design issues such as how to support the textual representation of multiple parallel threads of commands in what turned out to be a very limited graphical space, temporarily forced the design team back to pen and paper. Eventually, a final Visual Basic-based design sketch was produced.

While the Visual Basic-based design sketches did not have any functionality, screen dumps modified in Adobe Photoshop were used in the third design iteration to produce a Shockwave-based prototype in Macromedia Director showing a possible sequence of communication. Adding life to the Visual Basic-based design sketches in this "quick-and-dirty" fashion facilitated further discussions and resulted in minor modifications of the design before doing any actual programming.

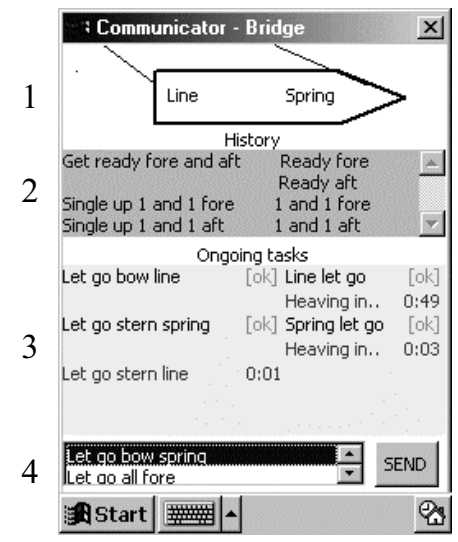

Figure 6. The user interface of the prototype

Finally, an experimental prototype was developed. The setup consisted of three iPAQs connected through a wireless network. One device was intended for the captain on the bridge while the other two were intended for 1 st and 2 nd officers on the fore and aft decks.

\subsection{User Interaction Design}

In order to avoid accidents in high-risk domains, understanding the state of the system operated or domain controlled is critical (Andersen, 2002). Consequently, Rasmussen (1983, 1986) suggest that computer interfaces should be designed to facilitate operators' reasoning about the domain of operation better and thus support human interaction which is contrary to total system automation as also discussed by Norman (1990).
Meeting these suggestions, we designed an interface that makes conversations persistent by providing the user with four sections that resemble the tense of conversations, cf. Figure 6:

1. Pictogram of the ship and mooring lines (present)

2. List of completed communication threads (past)

3. List of ongoing threads of communication (present)

4. List of unexecuted commands (future)

At the bottom of the screen unexecuted pre-defined commands and pending confirmations are displayed on a list (see Figure 6.4). The order of the list corresponds to the standard sequence of the overall operation, and utterances only appear when appropriate at the specific location of the device (bridge, fore or aft). By default, the most likely next step of the operation is highlighted. The list can be browsed and an utterance can be executed. It is then removed from the list and the most likely next utterance is highlighted.

As suggested in the discussion above, executing (present) conversations are sorted by means of objects rather than sequence. The representation of each conversation furthermore reflects the five stages in the conversation model.

When a command is given (a request), it appears in red on the list of ongoing threads of communication representing uncompleted tasks (see Figure 7.a). Next to it, a counter displays the time passed while waiting for confirmation. When a command is confirmed by repeating it back to the captain (a promise) it turns black and the timer is substituted by the text "[ok]" followed by a description of the current activity, e.g. "Singling up..." in red (see Figure 7.b). A counter next to this displays the time passed since confirmation (figure 6b). When a task is reported completed (an assert), a short statement in green (e.g. " 1 and 1 fore") substitutes the description of activity and the captain is prompted for confirmation (see Figure 7.c). When the completion of a task is confirmed by repeating it back to the deck (a declare) this is indicated by the text "[ok]" in green (see Figure 7.d). Completion of tasks, which automatically initiates a new task (e.g. heaving in lines when they are let go) results in an additional line of text displaying the current activity in the corresponding thread of communication.

When the captain confirms the completion of a task, the corresponding thread of communication is removed from the list of ongoing tasks and added at the bottom of the history list (see Figure 6.2). Hence it 
changes from present to past tense. The history list is simplified by removing less relevant and implicit information such as timers and confirmations (promises and declarations). When the history list is full, it automatically scrolls the oldest threads out of immediate sight.

(a)

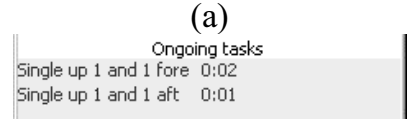

(b)

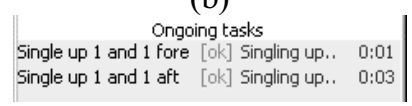

(c)

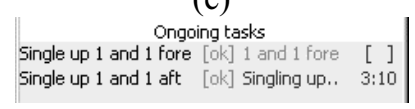

(d)

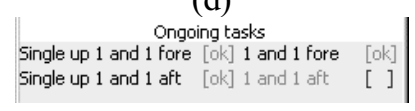

Figure 7. Commands being executed (a), confirmed (b), completed (c) and confirmed (d)

Generated from the formalized outcome of past and present threads of communication, a simple pictogram at the top of the screen graphically represents the lines attached to the quay for quick reference (see Figure 6.1).

\section{Validating the Conversation Model}

We conducted a usability evaluation to validate the design of the prototype and the underlying conversation model. The evaluation was user based.

\subsection{User-Based Evaluation}

The user-based evaluation took place in a state-ofthe art ship simulator imitating the operation of a large vessel in challenging weather and traffic conditions corresponding to a real world situation observed in the field studies (Nielsen, 2000).

Three teams of two trained maritime officers were given the task of letting go the lines and departing from harbour using the mobile system for communication between bridge and deck. One user acted as captain on the simulated bridge (see Figure 8) while the other acted as 1st officer on the fore deck in the neighbouring simulator control room. For simplicity, commands targeted at the 2nd officer on the aft deck were fed directly into the simulation and feedback was given by the simulation operator.

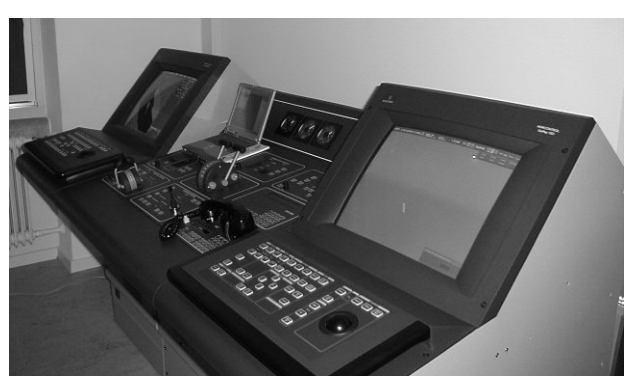

Figure 8. The ship simulator bridge

Carrying out the operation, the user acting as captain had to consider all aspects of manoeuvring the ship. This included controlling the rudder, propellers and thrusters as well as communicating with personnel on the ship, harbour traffic control etc. and taking into consideration the movements of other vessels.

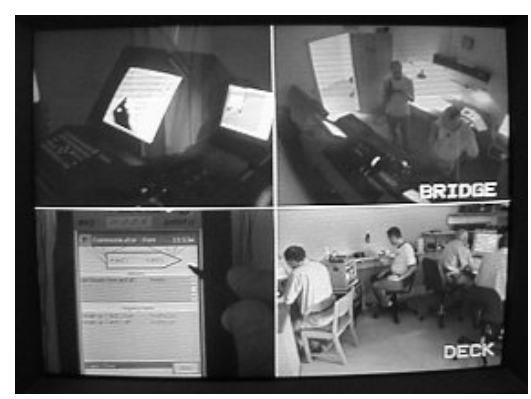

Figure 9. Video recording of use in the ship simulator

The primary task of the 1st officer on deck was to orally forward commands given by the captain via the mobile device prototype to the operator of the simulation (impersonating as the team of assistants carrying out the actual tasks) and report back to the captain. The operator would then enter the commands into the simulation, and report to the 1 st officer when the requests (such as letting go a line) had been carried out.

During the evaluation, the users were asked to think-aloud, explaining their experience of and interaction with the prototype. Two evaluators located on bridge and deck observed the users and asked questions for clarification. Following each evaluation session, a group interview of 10-15 minutes was carried out.

Merged signals from four cameras providing overviews of the bridge and deck/simulator control room as well as close-up views of the mobile devices were recorded on video (see Figure 9). Audio from the two rooms was recorded on separate tracks for possible separation during playback for analysis. 


\subsection{Results from the Evaluation}

The user-based evaluation provided rich usability data. Through detailed analysis of the videotapes we identified aspects of the design, which were experienced positively or negatively by the users and generated a list of usability problems experienced by the users.

The user-based evaluation showed that it was possible to communicate primarily by means of predefined text messages while doing a real-world operation. Moreover, the users expressed that the textbased interface gave them a simple channel of communication in which they could easily overview the ongoing communication and progress of the operation. The differentiation between future and present commands appeared straightforward as well as the grouping and progress of ongoing conversations. For quick overview, the small pictogram with a graphical representation of the ship was highly appreciated, and wishes for more details were expressed. The history list was rarely used.

The evaluation revealed a number of usability problems. This reflected that the current design of the mobile device did not support the handling of three types of non-standard situations. The first is retraction of a command. The captain may want to modify or withdraw a command that has already been issued. The second is error recovery. The change from continuous and open radio communication to discrete and closed text-based communication seems to introduce a risk of error and reduce the ability to recover from this. Wrong interaction as well as errors in conducting the tasks may arise and must be possible to recover from. The third type is unanticipated communication. In an emergency situation, communication changes from asynchronous to immediate because the situation develops quickly. In this situation, communicating unconstrained from pre-defined messages will be necessary.

These problems show that the decision to implement only a sub-set of the conversation for action model (Winograd and Flores, 1986) turned out to limit the usability of the design. Thus all users at some point of the operation decided to call the other one on the radio. In one situation, for example, a wrong command was executed due to interaction problems and could not be withdrawn. Therefore, the captain had to correct the error over the radio. In another situation, the ship had been brought into a situation, in which the remaining line was about to break. The officer on deck was aware of this problem, but had no means of reporting it textually to the captain.
Facilities for supporting this communication could easily be included in the system by extending the underlying model of communication structure.

\section{Conclusion}

The usability evaluation revealed promising results for the prototype. It was obvious that the problems of poor sound quality and lack of persistence were eliminated, and partial automation by suggesting commands proved possible. Furthermore, the graphical representation of the operation successfully supported maintenance of common ground. This indicates that the idea of using computer to make conversations persistent is relevant in this application domain and that the conversation model is a useful basis for design.

The usability evaluations and discussions with prospective users and domain experts also uncovered fundamental problems. Some of these can be handled within the complete conversation for action model. However, other problems that occur in unexpected situations emphasize a need for more dynamic conversations, and it will be difficult to support them. More work is needed to extend the model and identify fundamental limitations to persistent conversations.

\section{Acknowledgments}

We want to thank Peter Bøgh Andersen and Morten Nielsen who made their material available to us. We also want to thank Maersk Line for providing a setting for the study. The project in which this research was carried out is partly financed by the Danish Research Councils (grant number 2106-040022).

\section{References}

Andersen, P. B. (2000). Communication and Work on Maritime Bridges. CHMI Research report CHMI-1-2000 http://www.cs.auc.dk/ pba/ElasticSystems

Andersen, P. B. and May, M. (2001). Tearing up Interfaces. In Liu, K. et al (eds.): Information, organization and technology. Studies in organizational semiotics. Boston: Kluwer.

Camp, P. J., et al. (2000): Supporting Communication and Collaboration Practices in Safety-Critical Situations. In Proceedings of CHI'2000. New York: ACM Press, pp. 248250.

Computer Supported Cooperative Work (1994): vol. 2, no. 3 . 
Clark, H.H. and Schaefer, E. F. (1989): Contributing to discourse. Cognitive Science, vol. 13, pp. 259-294.

Divitini, M. (1999): Coordinating Cooperative Work: A Framework for the Design of Flexible Computer-Based Support. Aalborg University: Department of Computer Science, (PhD dissertation).

Erickson, T. and Herring, S. (2004) Persistent Conversation: A Dialog Between Research and Design. In Proceedings of HICSS-37. IEEE.

Fields, R. Paterno et al. Comparing Design and Options for Allocating Communication Media in Cooperative SafetyCritical Contexts: A Method and a Case Study. ACM TOCHI 6, 4 (1999), 370-398.

Heath, C. and Luff, P. (1992): Collaboration and Control. Crisis Management and Multimedia Technology in London Underground Line Control Rooms. Computer Supported Cooperative Work, vol. 1, pp. 69-94.

Ladkin, P. B. (1998a). The Ariane 5 Accident: A programming Problem. University of Bielefeld, Article RVS-J-98-0. Available at http://www.rvs.unibielefeld.de/publications/

Ladkin, P. B. (1998b). Computer-Related Incidents with Commercial Aircrafts: A compendium of Resources. Reports, Discussion and Commentary. University of Bielefeld. Available at http://www.rvs.unibielefeld.de/publications/Incidents/

Mackay, W. E. (1999). Is Paper Safer? The role of Paper Flight Strips in Air Traffic Control. ACM TOCHI 6, 4 (1999), 311-340.

Nielsen, C. and Søndergaard, A. Designing for mobility. Proc. NordiCHI'00, Royal Institute of Technology (2000)

Nielsen, M. (2000) Letting go the lines: Departure from the Felixstowe harbor. CHMI Research report CHMI-4-2000 http://www.cs.auc.dk/ pba/ElasticSystems
Norman, D. The 'Problem' With Automation: Inappropriate Feedback And Interaction Not Over-automation. In Broadbent D.E. et al. (eds.) Human Factors In Hazardous Situations, Oxford, Clarendon Press, 1990, 137-145.

Palanque, P. Paterno, F. and Wright, P. (1998). Designing User Interfaces for Safety-critical Systems. In proceedings of the conference on CHI 98 summary. Los Angeles, USA, ACM Press.

Patterson, E. S., Watts-Perotti, J. and Woods, D. D. (1999): Voice Loops as Coordination Aids in Space Shuttle Mission Control. Computer Supported Cooperative Work, vol. 8, pp. 353-371.

Perrow, C. (1984): Normal Accidents. Living with HighRisk Technologies. New York: Basic Books.

Popolov, D. Callaghan, M. and Luker, P. Conversation Space: Visualising Multi-threaded Conversation. Proc. AVI'00, ACM Press (2000).

Redmil, F. and Rajan, J. (1996). Human Factors in SafetyCritical Systems. Butterworth-Heinemann.

Schmidt, K. and Simone, C. (1996): Coordination Mechanisms: Towards a Conceptual Foundation of CSCW Systems Design. Computer Supported Cooperative Work, vol. 5, pp. 155-200.

Twitchell, D. P. and Nunamaker, J. F. (2004) Speech Act Profiling: A Probabilistic Method for Analyzing Persistent Conversations and Their Participants. In Proceedings of HICSS-37. IEEE.

van den Anker, F. W. G. and Lichtveld, R. A. (2000). Early Evaluation of New Technologies: The Case of Mobile Multimedia Communications For Emergency Medicine. In Vincent C. and de Mal B (eds.) Safety in Medicine. Qxford: Elsewier Science.

Winograd, T. and Flores, F. Understanding Computers and Cognition. Addison-Wesley, 1986. 